\title{
The Contribution of Decreased
} Muscle Size to Muscle Weakness in Children With Spastic Cerebral Palsy

\author{
Britta Hanssen 1,2, Nicky Peeters, 1,2, Ines Vandekerckhove ${ }^{1}$, Nathalie De Beukelaer ${ }^{1}$, \\ Lynn Bar-On 1,2,3, Guy Molenaers ${ }^{4,5}$, Anja Van Campenhout ${ }^{4,5}$, Marc Degelaen ${ }^{6,7,8}$, \\ Christine Van den Broeck ${ }^{2}$, Patrick Calders ${ }^{2}$ and Kaat Desloovere ${ }^{1,9 *}$
}

\begin{abstract}
${ }^{1}$ Department of Rehabilitation Sciences, Katholieke Universiteit (KU) Leuven, Leuven, Belgium, ${ }^{2}$ Department of Rehabilitation Sciences, Ghent University, Ghent, Belgium, ${ }^{3}$ Department of Rehabilitation Medicine, Amsterdam University Medical Center (UMC), Amsterdam, Netherlands, ${ }^{4}$ Department of Development and Regeneration, Katholieke Universiteit (KU) Leuven, Leuven, Belgium, ${ }^{5}$ Orthopaedic Section, University Hospitals Leuven, Leuven, Belgium, ${ }^{6}$ Department of Rehabilitation Research, Vrije Universiteit Brussel, Brussels, Belgium, ${ }^{7}$ Inkendaal Rehabilitation Hospital, Vlezenbeek, Belgium, ${ }^{8}$ University Hospital, Vrije Universiteit Brussel, Brussels, Belgium, ${ }^{9}$ Clinical Motion Analysis Laboratory, University Hospitals Leuven, Leuven, Belgium
\end{abstract}

Muscle weakness is a common clinical symptom in children with spastic cerebral palsy (SCP). It is caused by impaired neural ability and altered intrinsic capacity of the muscles.

OPEN ACCESS

Edited by:

Antigone Papavasiliou,

IASO General Hospital, Greece

Reviewed by:

Jessica Rose,

Stanford University, United States Christos Panteliadis,

Aristotle University of

Thessaloniki, Greece

*Correspondence:

Kaat Desloovere

kaat.desloovere@kuleuven.be

Specialty section: This article was submitted to

Pediatric Neurology, a section of the journal

Frontiers in Neurology

Received: 08 April 2021 Accepted: 15 June 2021

Published: 26 July 2021

Citation:

Hanssen B, Peeters N, Vandekerckhove I, De Beukelaer N,

Bar-On L, Molenaers G, Van Campenhout A, Degelaen M, Van den Broeck C, Calders P and Desloovere K (2021) The Contribution of Decreased Muscle Size to Muscle Weakness in Children With Spastic Cerebral Palsy.

Front. Neurol. 12:692582.

doi: 10.3389/fneur.2021.692582
To define the contribution of decreased muscle size to muscle weakness, two cohorts were recruited in this cross-sectional investigation: 53 children with SCP [median age, 8.2 (IQR, 4.1) years, 19/34 uni/bilateral] and 31 children with a typical development (TD) [median age, 9.7 (IQR, 2.9) years]. Muscle volume (MV) and muscle belly length for $\mathrm{m}$. rectus femoris, semitendinosus, gastrocnemius medialis, and tibialis anterior were defined from three-dimensional freehand ultrasound acquisitions. A fixed dynamometer was used to assess maximal voluntary isometric contractions for knee extension, knee flexion, plantar flexion, and dorsiflexion from which maximal joint torque (MJT) was calculated. Selective motor control (SMC) was assessed on a 5-point scale for the children with SCP. First, the anthropometrics, strength, and muscle size parameters were compared between the cohorts. Significant differences for all muscle size and strength parameters were found $(p \leq 0.003)$, except for joint torque per MV for the plantar flexors. Secondly, the associations of anthropometrics, muscle size, gross motor function classification system (GMFCS) level, and SMC with MJT were investigated using univariate and stepwise multiple linear regressions. The associations of MJT with growth-related parameters like age, weight, and height appeared strongest in the TD cohort, whereas for the SCP cohort, these associations were accompanied by associations with SMC and GMFCS. The stepwise regression models resulted in ranges of explained variance in MJT from 29.3 to $66.3 \%$ in the TD cohort and from 16.8 to $60.1 \%$ in the SCP cohort. Finally, the MJT deficit observed in the SCP cohort was further investigated using the TD regression equations to estimate norm MJT based on height and potential MJT based on MV. From the total MJT deficit, 22.6-57.3\% could be explained by deficits in MV. This investigation confirmed the disproportional decrease in muscle size and muscle strength around the knee and ankle joint in children with SCP, but also highlighted the large variability in the contribution of muscle size to muscle weakness.

Keywords: cerebral palsy, muscle weakness, muscle size, ultrasound, muscle volume, selective motor control 


\section{INTRODUCTION}

Cerebral palsy (CP) describes a group of permanent disorders of the development of movement and posture, causing activity limitation, that are attributed to non-progressive disturbances that occurred in the developing fetal or infant brain. It is the most common cause of childhood-onset physical disability (1). Spastic CP (SCP) is the largest subcategory, affecting between 70 and $80 \%$ of children with CP (2). Children with SCP present with physical impairments like abnormal gait and gross motor function, which can deteriorate gradually. These physical impairments are associated with limitations in daily life activities and restrictions in societal participation. They are primarily caused by neural impairments including spasticity, decreased selected motor control (SMC), and poor postural stability. Additionally, the neural impairments can lead to secondary nonneural musculoskeletal impairments like altered intrinsic muscle structure, muscle contractures, and bony deformities (3).

Another consistent clinical finding in children with SCP is muscle weakness (4-7), defined as an inability to produce or maintain an anticipated level of force (8). When studying mechanisms underlying physical disability, higher associations with gross motor function have been reported for strength and selectivity than for spasticity (9-13). In ambulant children with SCP, lower extremity muscle strength deficits have been identified, ranging from 15 to $80 \%$, with larger deficits reported for less functional children $(4,5,14)$. Moreover, the increase in strength during growth is lower than in typically developing (TD) children and suggested to be insufficient in relation to increases in body mass $(15,16)$.

However, muscle weakness cannot be categorized as just a neural or just a musculoskeletal impairment. Both the neural ability to selectively activate the muscle and the intrinsic capacity of the muscle influence strength production $(6,7)$. Furthermore, the assessment of strength in children with SCP can also be influenced by cognitive, attentional, or motivational difficulties (17). The primary cause of muscle weakness in children with SCP is impaired neural function due to damage to the descending pathways of the central nervous system (7). The central damage impacts the ability to maximally activate the agonists and the degree of cocontraction of antagonists (6, $7,18,19)$. Secondarily, muscle weakness is caused by intrinsic muscle property alterations involving decreases in muscle size, deteriorated muscle integrity, and potentially also changes in fascicle arrangement $(17,20-22)$.

Improved access to muscle imaging techniques in clinical research has led to a great increase in the number of studies quantifying intrinsic muscle properties in children with SCP (23). Lower limb muscle volume (MV) reduction ranging from 18 to $50 \%$ has been documented $(6,24-31)$. Similar reductions have been described based on two-dimensional ultrasound (US) measures of muscle size like muscle thickness and cross-sectional area $(\mathrm{CSA})(6,17,29,32,33)$. These deficits in muscle size present as early as the age of 15 months $(27,34,35)$ and increase into later childhood and adolescence (36). Comparable to muscle weakness, muscle size is also related to the level of functionality, with larger reductions seen in less functional children $(25,26,29,37-39)$. However, large variability has been reported between subjects and between different muscles, often with distal predominance of MV reduction $(25,28)$.

The physiological CSA (pCSA) of a muscle is the ratio of MV to fascicle length. This value represents the number of fascicles in parallel. It is therefore directly related to the force-generating capacity of a muscle. Since pCSA and muscle size show a strong association with muscle strength in TD populations $(40,41)$, it can serve as a proxy measure of potential strength in children with SCP. It is likely that decreased MV and shorter or similar fascicle lengths result in decreased PCSA in children with SCP $(29,42)$. However, as discussed above, the decrease in muscle strength is larger than the reduction in MV. While investigating the muscle size-strength relationship, Reid et al. (20) found a weaker association between knee flexors and extensors MV and anatomical CSA (aCSA) and joint torque in comparison to TD children. Elder et al. (6) found a reduction in specific tension, defined as the ratio of joint torque and aCSA, for both the plantar and dorsiflexors in SCP. The disproportional decrease in muscle strength could be related to other properties such as impaired neural control $(6,7)$.

Most focal treatments for children with SCP, like strength training, casting, and botulinum neurotoxin-A injections, are administered at the muscular level $(43,44)$. Knowing the contribution of decreased muscle size to muscle weakness is of importance in choosing the appropriate treatment options. The muscle size-strength relationship in children with SCP has been evaluated both at the knee and the ankle, but in different, possibly not equivalent, ways $(6,20)$. Additionally, in investigations about the factors underpinning gross motor function, parameters of muscle size have been combined with neuromotor symptoms like SMC and indirect estimates of muscle quality $(12,24)$. Evaluating the muscle size-strength relationship at the knee and ankle joint in one cohort of children with SCP, using data of TD children as a reference, as well as including SMC, could improve our understanding of the neural and non-neural contributions to muscle weakness in this population.

The first aim of this investigation was to describe the deficits in muscle strength, muscle size, and their ratio for knee flexors and extensors, plantar, and dorsiflexors in children with SCP. Secondly, the muscle size-strength relationship was defined, combined with the influence of age, anthropometric measures, functional level, and SMC following a multiple linear regression approach. Finally, the contribution of muscle weakness due to decreased muscle size to the total deficit in muscle strength was defined for the four investigated joint movements.

\section{MATERIALS AND METHODS}

\section{Participants}

This investigation included a convenience sample of 84 participants, 53 with SCP (19 unilateral and 34 bilateral) and a control group of $31 \mathrm{TD}$ children, who were recruited at the Clinical Motion Analysis Laboratory at the University Hospitals Leuven, Belgium. Inclusion criteria for the SCP cohort were a confirmed diagnosis of SCP, age between 5 and 12 years, and 
gross motor function classification system (GMFCS) level IIII (45). Botulinum neurotoxin-A injections 6 months prior to the assessments, lower limb bony surgical interventions 2 years prior to the assessments, and history of lower limb muscular surgeries at any time point were defined as exclusion criteria. In case of insufficient cooperation during testing or insufficient understanding of the test procedures, the participant was also excluded. TD children were recruited via hospital co-workers and students within the same age range of 5-12 years and could not have any known neurological or orthopedic lower limb problems. All data were collected as part of an ongoing project that was approved by the local Ethical Committee of the University Hospitals Leuven (S59945) and Ghent (EC/2017/0526). Written informed consent was obtained from all parents or caregivers.

\section{Data Collection and Analysis}

\section{Participant Characteristics and Anthropometrics}

The clinical and anthropometric features of the participants are summarized in Table 1. The most affected leg was assessed in the participants with SCP, according to the clinical reports and the most recent clinical exam results for muscle spasticity (Modified Ashworth Scale and Modified Tardieu angle) and strength (Medical Research Council grade scale) at the knee and ankle (46-48). If the clinical examination indicated no difference, the assessed leg for study was chosen at random by flipping a coin. In the TD cohort, the assessed leg was identified in the same way. Age, weight, and height were recorded for each participant. A clinical classification of SMC assessed in standard manual muscle testing position, graded on a 5-point scale between 0 and 2, was used for knee extension (KE), knee flexion (KF), plantar flexion $(\mathrm{PF})$, and dorsiflexion (DF) [adapted from the method described by Gage et al. (49)].

\section{Three-Dimensional Freehand Ultrasonography}

Three-dimensional freehand ultrasonography (3DfUS) acquisitions were performed by combining a conventional two-dimensional B-mode ultrasonography device (TelemedEchoblaster 128 Ext-1Z, with a $5.9-\mathrm{cm} 10-\mathrm{MHz}$ linear US transducer, Telemed Ltd., Vilnius, Lithuania) with a motion tracking system (Optitrack V120:Trio, NaturalPoint Inc., Corvallis, Oregon, USA) (50). According to a previously described technique, four markers were attached to the US probe and tracked by the motion tracking system, resulting in the synchronized position and orientation of every acquired two-dimensional US image (50).

Both data collection and processing were performed using STRADWIN software (version 6.0; Mechanical Engineering, Cambridge University, Cambridge, UK) for four lower limb muscles: $\mathrm{m}$. rectus femoris (RF), $\mathrm{m}$. semitendinosus (ST), $\mathrm{m}$. tibialis anterior (TA), and $\mathrm{m}$. gastrocnemius medialis (MG). The reliability of 3DfUS has been confirmed for the plantar flexor muscles (50-52), as well as for the processing of the TA, RF, and ST (53). MV (in milliliters) was estimated by drawing equally spaced transverse plane segmentations along the inside of the muscle border for $\sim 5 \%$ of all acquired images, followed by an automatic linear interpolation. The reconstructed muscle was visually inspected, and additional images were segmented to improve the interpolated shape if needed. Muscle length (ML) (in millimeters) was determined as the linear distance between muscle origin and distal muscle tendon junction. MV was normalized to body mass ( $\mathrm{nMV} ; \mathrm{ml} / \mathrm{kg}$ ) and $\mathrm{ML}$, to subject height ( $\mathrm{nML} ; \mathrm{mm} / \mathrm{cm}$ ), enabling comparisons between cohorts. More details about the measurement and processing protocol can be found in Supplementary File 1.

\section{Isometric Strength Assessments}

Maximum voluntary isometric contractions (MVIC) were collected for KE, KF, PF, and DF with a fixed dynamometer (MicroFet 2, Hogan Health Industries, West Jordan, Utah, USA) in a previously described, custom-designed chair (5). The procedure is further explained in Supplementary File 2. Custom-written MATLAB scripts were used to determine the peak force (in Newton) of each MVIC, from which average maximal joint torque (MJT; Nm) and normalized joint torque (nMJT; Nm/kg) were calculated over the three MVIC trials (5).

\section{Statistical Analysis}

Data were analyzed using SPSS (Version 26, SPSS Inc., Chicago, Illinois, USA). A ratio of strength to muscle size was calculated for every joint movement by dividing the MJT by the corresponding MV. Percentage differences in muscle strength and muscle size between TD and SCP were calculated as shown below, where $\mathrm{M}$ represents the median of the specified parameter:

$$
\% \text { Diff }=\left(\frac{M_{S C P}-M_{T D}}{M_{T D}}\right) * 100 \%
$$

A negative percentage indicated a deficit in the SCP group in comparison to the TD group. Normality of the data distribution was evaluated using the Shapiro-Wilk test, histograms, and QQ plots. Since most parameters were not normally distributed, all descriptive statistics are presented as median (interquartile range). Bonferroni corrections were applied for multiple testing and specified beneath each table.

To investigate the first aim, differences between the TD and SCP cohort were assessed using a Student's $t$-test (after confirming equality of variances) or the Mann-Whitney $U$-test.

For the second aim, linear associations between anthropometric measures (normalized) MV and ML, SMC, GMFCS, and (n)MJT were explored by univariate linear regression. Standardized residuals ( $\geq 3$ standard deviations) were used to remove outliers, and normal distribution of residuals and heteroscedasticity were checked. Correlation coefficients were classified as negligible $(r<0.300)$, low $(r=0.300-0.499)$, moderate $(r=0.500-0.699)$, high $(r=0.700-0.899)$, or very high $(r \geq 0.900)$ (54), and differences in correlation coefficients between TD and SCP were defined by Fisher $Z$-scores. Based on the linear association with (n)MJT and the interassociations of potential independent variables within the categories anthropometrics (age, weight, and height), muscle morphology (MV and ML), and clinical scales (SMC and GMFCS), one parameter was selected per category to be used in the multivariate analyses. Multiple linear regression models were built using a backward approach (enter $p \leq 0.05$, remove $p \geq 0.10$ ). A first 
TABLE 1 | Participant characteristics.

\begin{tabular}{|c|c|c|c|c|c|c|}
\hline & \multicolumn{2}{|c|}{$\begin{array}{l}\text { Typically developing } \\
\qquad(n=31)\end{array}$} & \multicolumn{2}{|c|}{$\begin{array}{l}\text { Spastic cerebral palsy } \\
\qquad(n=53)\end{array}$} & \multicolumn{2}{|c|}{ Comparison } \\
\hline & Median & IQR & Median & IQR & $T$-test & MWU \\
\hline Age (yrs) & 9.7 & 2.9 & 8.2 & 4.1 & & 0.064 \\
\hline Weight (kg) & 29.2 & 9.1 & 27.3 & 13.6 & & 0.407 \\
\hline \multirow[t]{2}{*}{ Height (cm) } & 138.6 & 17.5 & 130.4 & 19.3 & 0.021 & \\
\hline & \multicolumn{2}{|c|}{ Frequencies } & \multicolumn{2}{|c|}{ Frequencies } & & \\
\hline $\operatorname{Sex}(F / M)$ & \multicolumn{2}{|c|}{$16 / 15$} & \multicolumn{2}{|c|}{ 22/31 } & & \\
\hline Involvement (uni/bi) & \multicolumn{2}{|c|}{ NA } & \multicolumn{2}{|c|}{$19 / 34$} & & \\
\hline GMFCS (I/II/III) & \multicolumn{2}{|c|}{ NA } & \multicolumn{2}{|c|}{$32 / 12 / 9$} & & \\
\hline \multicolumn{7}{|c|}{$\operatorname{SMC}(2 / 1.5 / 1 / 0.5 / 0)(n=52)$} \\
\hline Knee extension & \multicolumn{2}{|c|}{ NA } & \multicolumn{2}{|c|}{ 33/13/6/0/0 } & & \\
\hline Knee flexion & \multicolumn{2}{|c|}{ NA } & \multicolumn{2}{|c|}{ 24/15/7//0/1 } & & \\
\hline Plantar flexion & \multicolumn{2}{|c|}{ NA } & \multicolumn{2}{|c|}{$18 / 15 / 7 / 4 / 4$} & & \\
\hline Dorsiflexion & \multicolumn{2}{|c|}{ NA } & \multicolumn{2}{|c|}{ 23/20/9/6/1 } & & \\
\hline
\end{tabular}

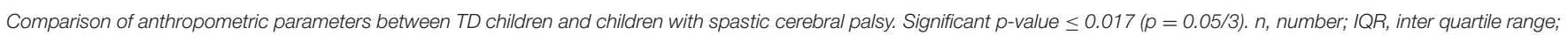
MWU, Mann-Whitney U test; F, female; M, male; NA, not applicable; GMFCS, gross motor function classification system; SMC, selective motor control.

model included the same parameters for both cohorts from the categories anthropometrics and muscle morphology. In a second model for the SCP cohort, the additional explained variance by a clinical scale was explored. Standardized residuals and Cook's distance value were used to diagnose and remove outliers. Final models were assessed for normal distribution of residuals, multicollinearity, and heteroscedasticity.

For the third aim, the MJT deficit observed in the SCP cohort was further investigated using the TD regression equations. The regression equation of MV with MJT defined in the TD cohort was used to estimate potential muscle strength in children with SCP (MJT $\mathrm{T}_{\text {potential }}$ ) based on their MV. The regression equation for MJT based on an anthropometric variable was used to estimate the expected norm value of muscle strength for children with SCP $\left(\mathrm{MJT}_{\text {norm }}\right)$. Muscle strength profiles based on the relative contribution of decrease in $\mathrm{MV}\left(\mathrm{MJT}_{\text {deficitMV }}\right)$ and the other factors $\left(\mathrm{MJT}_{\text {deficitother }}\right)$ to muscle weakness were calculated as a percentage of the total strength deficit (Figure 2A).

There were some missing data due to 3DfUS reconstructions that could not be analyzed, MVICs that could not be assessed, and missing information about SMC. For the univariate linear regressions, the participant with missing data was excluded for all analyses of the specific joint movement. An overview of missing data is added in Supplementary Table 1.

\section{RESULTS}

Like in the Methods section, the results are structured in the order of the three aims. First, the descriptive results and deficits of SCP children in comparison to TD children are described and reported in Tables 1, 2. Thereafter, the linear associations and multiple regression models are discussed and presented in
Figure 1 and Tables 3, 4. Finally, the contributions to muscle weakness are explored and reported in Figure 2.

\section{Deficits}

Descriptive results of participant characteristics are shown in Table 1. There were no significant differences in age, weight, and height between the SCP and TD groups. Table 2 describes the differences in muscle morphology and strength parameters between the TD children and children with SCP. Both MV and $\mathrm{nMV}$ for all four muscles were significantly decreased in the SCP cohort $(p \leq 0.002)$. For nMV, the median deficits ranged between $19.7 \%$ for the ST and $43.5 \%$ for the TA. Similarly, both ML and nML were significantly decreased in the SCP cohort for all four muscles except for nML of the RF, which was close to significance $(p \leq 0.011)$. Differences in median $\mathrm{nML}$ ranged from $4.6 \%$ for the RF to $11.3 \%$ for the MG. Similar to the alterations in muscle morphology, both MJT and nMJT were significantly decreased for all four joint movements $(p<0.001)$. Normalized MJT showed median decreases ranging from $47.1 \%$ for PF to $71.7 \%$ for DF. The ratio of joint torque over muscle size was also significantly decreased for $\mathrm{KE}, \mathrm{KF}$, and $\mathrm{DF}$, with median deficits of 40.3-54.0\% ( $p<0.001)$, but PF showed a lower, non-significant deficit of $18.1 \%(p=0.071)$.

\section{Relationships}

The univariate associations of anthropometric parameters, muscle morphology, GMFCS, and SMC with MJT, as well as with nMJT, are depicted in Table 3. In the TD cohort, all anthropometric and muscle morphology parameters showed significant moderate to high associations with MJT of KE, KF, and DF $(r=0.537-0.849, p \leq 0.003)$. For PF, only age and height showed significant moderate associations $(r=0.502-0.556, p$ $\leq 0.008)$. Associations of PF with weight and MV were close to significance but low ( $r=0.449-0.463, p \leq 0.019)$. Similarly, 
TABLE 2 | Comparisons of muscle morphology and maximal joint torque.

\begin{tabular}{|c|c|c|c|c|c|c|c|c|c|}
\hline & \multicolumn{3}{|c|}{ Typically developing } & \multicolumn{3}{|c|}{ Spastic cerebral palsy } & \multicolumn{3}{|c|}{ Comparison } \\
\hline & $n$ & Median & IQR & $n$ & Median & IQR & $T$-test & MWU & $\%$ \\
\hline \multicolumn{10}{|l|}{ Muscle volume (ml) } \\
\hline Rectus femoris & 31 & 96.0 & 32.0 & 53 & 64.9 & 33.2 & & $<0.001^{*}$ & -32.7 \\
\hline Semitendinosus & 29 & 68.8 & 31.3 & 48 & 48.8 & 30.6 & & $0.002^{\star}$ & -30.4 \\
\hline Medial gastrocnemius & 27 & 68.5 & 29.1 & 49 & 40.0 & 26.4 & & $<0.001^{\star}$ & -41.6 \\
\hline Tibialis anterior & 28 & 48.6 & 28.7 & 49 & 25.5 & 12.6 & & $<0.001^{*}$ & -47.6 \\
\hline \multicolumn{10}{|c|}{ Normalzied MV (ml/kg) } \\
\hline Rectus femoris & 31 & 3.17 & 0.61 & 53 & 2.30 & 0.63 & $<0.001^{\star}$ & & -27.5 \\
\hline Semitendinosus & 29 & 2.24 & 0.75 & 48 & 1.80 & 0.36 & $<0.001^{*}$ & & -19.7 \\
\hline Medial gastrocnemius & 27 & 2.44 & 0.41 & 49 & 1.49 & 0.76 & & $<0.001^{*}$ & -38.9 \\
\hline Tibialis anterior & 28 & 1.60 & 0.49 & 49 & 0.90 & 0.25 & & $<0.001^{*}$ & -43.5 \\
\hline \multicolumn{10}{|l|}{ Muscle length (mm) } \\
\hline Rectus femoris & 31 & 256.8 & 47.1 & 53 & 224.5 & 47.5 & $0.003^{\star}$ & & -12.6 \\
\hline Semitendinosus & 29 & 244.1 & 50.8 & 48 & 221.0 & 46.7 & $<0.001^{\star}$ & & -9.5 \\
\hline Medial gastrocnemius & 27 & 185.5 & 39.6 & 49 & 146.1 & 39.9 & & $<0.001^{*}$ & -21.3 \\
\hline Tibialis anterior & 28 & 242.9 & 58.0 & 49 & 198.2 & 43.7 & & $<0.001^{\star}$ & -18.4 \\
\hline \multicolumn{10}{|c|}{ Normalized muscle length (mm/cm) } \\
\hline Rectus femoris & 31 & 1.84 & 0.23 & 53 & 1.75 & 0.16 & & 0.011 & -4.6 \\
\hline Semitendinosus & 29 & 1.81 & 0.28 & 48 & 1.68 & 0.21 & $0.001^{*}$ & & -7.2 \\
\hline Medial gastrocnemius & 27 & 1.35 & 0.15 & 49 & 1.19 & 0.24 & & $<0.001^{*}$ & -11.3 \\
\hline Tibialis anterior & 28 & 1.74 & 0.23 & 49 & 1.58 & 0.20 & $<0.001^{*}$ & & -9.1 \\
\hline \multicolumn{10}{|c|}{ Maximal joint torque (Nm) } \\
\hline Knee extensors & 31 & 31.0 & 35.5 & 53 & 13.3 & 18.4 & & $<0.001^{*}$ & -57.1 \\
\hline Knee flexors & 29 & 22.5 & 11.9 & 48 & 6.7 & 10.4 & & $<0.001^{*}$ & -70.2 \\
\hline Plantar flexors & 27 & 12.5 & 8.3 & 49 & 7.0 & 5.2 & & $<0.001^{*}$ & -44.0 \\
\hline Dorsiflexors & 28 & 9.9 & 6.0 & 49 & 2.3 & 2.0 & & $<0.001^{\star}$ & -77.2 \\
\hline \multicolumn{10}{|c|}{ Normalized maximal joint torque ( $\mathrm{Nm} / \mathrm{kg})$} \\
\hline Knee extensors & 31 & 1.11 & 0.63 & 53 & 0.54 & 0.53 & & $<0.001^{\star}$ & -51.8 \\
\hline Knee flexors & 29 & 0.74 & 0.49 & 48 & 0.28 & 0.30 & & $<0.001^{\star}$ & -62.4 \\
\hline Plantar flexors & 27 & 0.42 & 0.21 & 49 & 0.22 & 0.24 & & $<0.001^{*}$ & -47.1 \\
\hline Dorsiflexors & 28 & 0.31 & 0.16 & 49 & 0.09 & 0.07 & & $<0.001^{*}$ & -71.7 \\
\hline \multicolumn{10}{|c|}{ Joint torque/muscle size (Nm/ml) } \\
\hline Knee extensors & 31 & 0.40 & 0.19 & 53 & 0.24 & 0.20 & & $<0.001^{*}$ & -40.3 \\
\hline Knee flexors & 29 & 0.31 & 0.16 & 48 & 0.14 & 0.17 & & $<0.001^{\star}$ & -54.0 \\
\hline Plantar flexors & 27 & 0.19 & 0.10 & 49 & 0.15 & 0.13 & & 0.071 & -18.1 \\
\hline Dorsiflexors & 28 & 0.19 & 0.11 & 49 & 0.09 & 0.06 & $<0.001^{\star}$ & & -51.2 \\
\hline
\end{tabular}

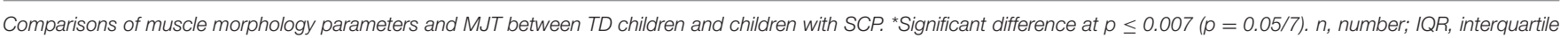
range; $M W U$, Mann-Whitney $U$-test.

in the SCP cohort, associations of anthropometrics and muscle morphology with MJT were significant for KE and KF, with coefficients ranging from low to high $(r=0.437-0.746, p \leq$ $0.007)$. For DF, height, $\mathrm{MV}$, and ML were significant, whereas age and weight were close to significance $(r=0.353-0.639, p$ $\leq 0.013$ ). Only MV and ML were significant for PF with low associations $(r=0.413-0.431, p \leq 0.003)$. GMFCS level showed a low significant association with $\mathrm{KE} \mathrm{MJT}$ and a low, close to significant association with $\mathrm{KF}(r=-0.340-0.469, p \leq 0.018)$, but negligible non-significant associations with PF and DF MJT $(r=-0.251, p \leq 0.082)$. SMC was moderately significantly correlated with KE and PF MJT $(r=0.450-0.480, p=0.001)$, close to significant for $\mathrm{KF}(r=0.337, p=0.021)$, and there was no association with DF MJT. Fisher's Z-scores did not demonstrate significant differences between the reported relationships for children with and without SCP, except for KE with weight $(Z=$ 2.442) and age with DF MJT $(Z=1.764)$.

To eliminate the influence of growth on the associations with MJT, the univariate analyses were also performed with normalized parameters (nMJT, nMV, and nML). In the TD cohort, only the association of weight and height with KE nMJT remained significant $(r=0.486-0.528, p \leq 0.006)$. However, in the SCP cohort, nMV associated significantly with nMJT of KE, PF, and DF ( $r=0.401-0.608, p \leq 0.004)$, and $\mathrm{nML}$ 
with KE nMJT ( $r=0.454, p=0.001)$. GMFCS showed low to moderate significant associations with $\mathrm{KE}$ and $\mathrm{KF}$ nMJT $(r=$ -0.504 to $-0.414, p \leq 0.003$ ), and SMC showed low to moderate significant associations with $\mathrm{KE}, \mathrm{KF}$, and PF $(r=0.442-0.528$, and $p \leq 0.002$ ).

Based on the univariate associations with MJT (Table 3) and the interassociations between predictors within each category (Supplementary Table 2), the following parameters were selected: height within the category anthropometrics, MV within muscle morphology, and SMC within clinical scales. The results of the multiple linear regression models for MJT in both cohorts are presented in Table 4. The models in the TD cohort (Table 4A) included height, MV, or both parameters. All regression models were significant $(p \leq 0.002)$, and explained variance ranged from $29.3 \%$ for PF to $66.3 \%$ for KE. The results in the SCP cohort for MJT (Table 4B) were also all significant $(p \leq 0.002)$, and in the first model, with the same independent variables as in the TD cohort, only MV was included per joint movement. Explained variance ranged from $16.8 \%$ for $\mathrm{PF}$ to $54.8 \%$ for KE. In the second model, also SMC was entered into the model. The model for DF MJT did not change, whereas SMC was included for KE, KF, and PF MJT. The regression coefficients increased accordingly, explaining an additional $5.3-11.1 \%$ of the variance compared to the first model (Table 4B). The multiple linear regression models in the SCP cohort for nMJT had explained variances ranging from 21.0 to $41.9 \%$ and were all significant $(p \leq 0.002)$ (Table 4C). The included parameters were SMC, nMV, or both.

\section{Contributions to Muscle Weakness}

Although the correlation coefficients of MV with MJT were similar between TD and SCP, the regression coefficients or slopes of this relationship tended to be lower in the SCP cohort for all joint movements, except for KF. However, there was a large difference in the regression constant for KF (Figure 1). The children with SCP are largely located at the bottom-left quadrant of the graphs, pointing toward lower MV with even lower MJT than what would be potential for the MV.

Figure 2 and Supplementary Table 3 show the relative deficits in MJT divided into the part caused by the decrease in $\mathrm{MV}\left(\mathrm{MJT}_{\text {deficitMV }}\right)$ and the part resulting from other factors, like decreased neural control or alterations in muscle composition ( $\left.\mathrm{MJT}_{\text {deficitother }}\right)$. $\mathrm{MJT}_{\text {deficittotal }}$ as a percentage of $\mathrm{MJT}_{\text {norm }}$ ranged from $44.7 \%$ for $\mathrm{PF}$ to $73.3 \%$ for $\mathrm{DF}$. The contribution of $\mathrm{MJT}_{\text {deficitMV }}$ was largest for $\mathrm{KE}$ with 57.3\%, followed by PF (47.7\%), DF (39.3\%), and KF (22.6\%). The muscle strength profiles for every participant are depicted in Supplementary Figure 1, indicating the absolute values of $\mathrm{MJT}_{\text {measured }}, \mathrm{MJT}_{\text {deficitMV}}$, and $\mathrm{MJT}_{\text {deficitother }}$. Supplementary Figure 1 shows that the contribution of decreased MV to muscle weakness is not constant, as it changes with increasing MJT. At the younger ages, there were some children who did not have a deficit based on MV, especially for $\mathrm{KF}$, and $\mathrm{KF}$ and $\mathrm{DF}$ showed an almost constant and large contribution of other factors to the $\mathrm{MJT}_{\text {deficittotal. However, the }}$ contribution of a deficit in MV appeared to increase with growth and therefore with increasing $\mathrm{MJT}_{\text {norm. }}$. Supplementary Figure 1 specifically highlights the high heterogeneity, not only between joint movements but also between subjects.

\section{DISCUSSION}

\section{Deficits}

The first aim of this investigation was to define the deficits in muscle strength, muscle size, and their ratio for KF, KE, PF, and DF in children with SCP. The children with SCP demonstrated significant deficits in MV compared to their TD peers, for all assessed muscles (Table 2). Since the TD cohort appeared slightly older (Table 1), MV was also normalized to body weight, but the differences remained. The deficits in $\mathrm{nMV}$, ranging from 19.7 to $43.5 \%$, were consistent with earlier results in literature $(6,24,25,28,30)$. The differences in deficits in proximal and distal muscles were also in line with previous findings, with the proximal muscles showing $\sim 70 \% \mathrm{MV}$ of the TD cohort values and the distal muscles showing $50-60 \% \mathrm{MV}$ of the values of TD children $(25,28)$. Similarly, ML and nML were significantly decreased, with deficits in $\mathrm{nML}$ ranging from 4.6 to $11.3 \%$. The decreases in muscle size parameters were accompanied with significant decreases in muscle strength, ranging from 47.1 to $71.7 \%$ for the nMJT (Table 2). These deficits are consistent with earlier reported deficits in muscle strength in children with $\mathrm{CP}$ $(4,5,15)$. In the current investigation, $\mathrm{KE}$ and $\mathrm{KF}$ were similarly weakened, whereas DF was far more affected than PF. This difference between DF and PF torque could be partly explained by the different impact of the ankle joint angle at which the strength was assessed, which was in neutral $\left(90^{\circ}\right)$. This joint angle might already have induced a stretch on the plantar flexors, which the dorsiflexor muscles first must overcome.

The disproportionality in the deficits in muscle strength and size in children with SCP was a first confirmation of an altered muscle size-strength relationship. This was further confirmed in the ratio of torque over size, which was significantly lower in the SCP group in comparison to the TD group for three of the four muscle groups. Both $\mathrm{KE}$ and KF, as well as DF, showed deficits in strength per muscle size of 40.3-54.0\%. Only $\mathrm{PF}$ was an exception, where the relative decrease in MV was almost equal to the relative decrease in MJT, resulting in a similar muscle strength-to-size ratio in the two cohorts. In the investigation by Elder et al. (6), the specific tension defined as torque over the whole muscle group CSA of both PF and DF was significantly reduced. However, they also found a few cases where torque was proportional to the CSA. The results for PF in the current investigation are in line with results by O'Brien et al. (55), who found that muscle activation capacity did not strongly predict ankle PF weakness in high-functioning adults with SCP, suggesting that muscle size may contribute more to weakness than neural voluntary activation. However, it should also be noted that PF is probably the joint movement where compensation from more proximal joints is most challenging to be avoided in the fixated position used in this investigation, as is also visible in some of the relatively high $\mathrm{MJT}_{\text {measured }}$ bars in Supplementary Figure 1. Despite the heterogeneity between the current and previous study results, these findings confirm that 

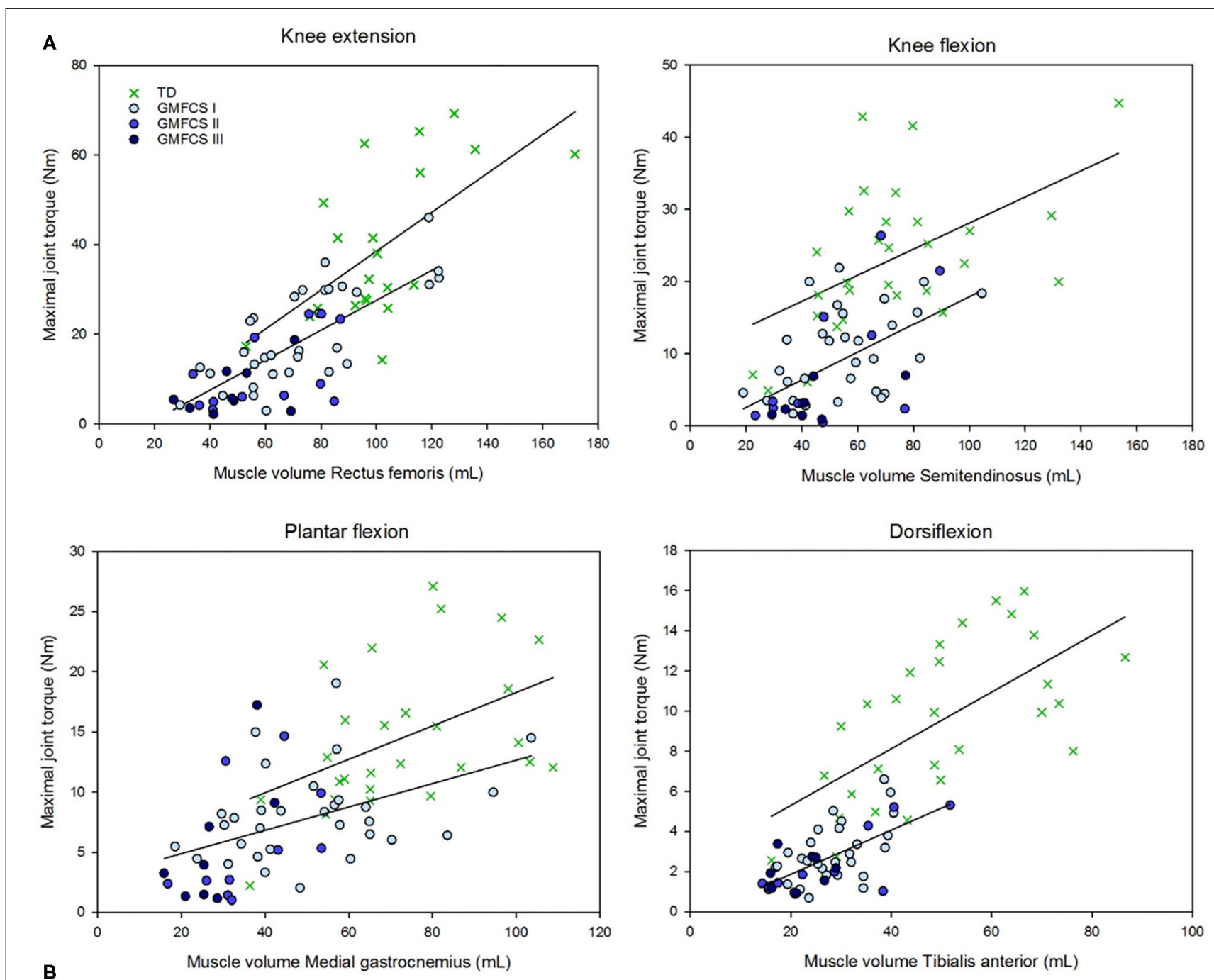

B

\begin{tabular}{|c|c|c|c|c|c|c|c|c|c|}
\hline \multirow[b]{2}{*}{ Dependent variable } & \multirow[b]{2}{*}{$\begin{array}{c}\mathbf{N} \\
\text { (outliers) }\end{array}$} & \multicolumn{3}{|c|}{ Constant } & \multicolumn{3}{|c|}{ Regression coefficient } & \multicolumn{2}{|c|}{$\mathbf{R}^{2}$} \\
\hline & & Value & SE & $p$-value & Value & SE & $\mathrm{p}$-value & Value & $p$-value \\
\hline \multicolumn{10}{|c|}{ Typically developing cohort } \\
\hline Knee extension & $31(0)$ & -11.179 & 7.566 & 0.15 & 0.527 & 0.072 & $<0.001$ & 0.648 & $<0.001$ \\
\hline Knee flexion & $29(0)$ & 10.021 & 4.258 & 0.026 & 0.181 & 0.055 & 0.003 & 0.262 & 0.003 \\
\hline Plantar flexion & $27(1)$ & 4.386 & 4.012 & 0.285 & 0.139 & 0.053 & 0.015 & 0.183 & 0.015 \\
\hline Dorsiflexion & $28(2)$ & 2.470 & 1.729 & 0.165 & 0.141 & 0.033 & $<0.001$ & 0.393 & $<0.001$ \\
\hline \multicolumn{10}{|c|}{ Spastic cerebral palsy cohort } \\
\hline Knee extension & $53(0)$ & -6.609 & 2.984 & 0.031 & 0.341 & 0.043 & $<0.001$ & 0.557 & $<0.001$ \\
\hline Knee flexion & $48(1)$ & -1.292 & 2.442 & 0.599 & 0.192 & 0.044 & $<0.001$ & 0.281 & $<0.001$ \\
\hline Plantar flexion & $49(0)$ & 2.645 & 1.504 & 0.085 & 0.105 & 0.032 & 0.002 & 0.168 & 0.002 \\
\hline Dorsiflexion & $49(2)$ & -0.334 & 0.544 & 0.542 & 0.110 & 0.019 & $<0.001$ & 0.396 & $<0.001$ \\
\hline
\end{tabular}

FIGURE 1 | Relationship between muscle volume and maximal joint torque for four lower limb joint movements in children with a typical development (TD) and with spastic cerebral palsy with gross motor function classification scale (GMFCS) levels I to III. (B) Regression formula parameters for the estimation of maximal joint torque based on muscle volume as shown in (A). SE, standardized error. The outliers indicate the cases that were removed based upon the standardized residuals or Cook's distance. 
TABLE 3 | Associations with maximal joint torque.

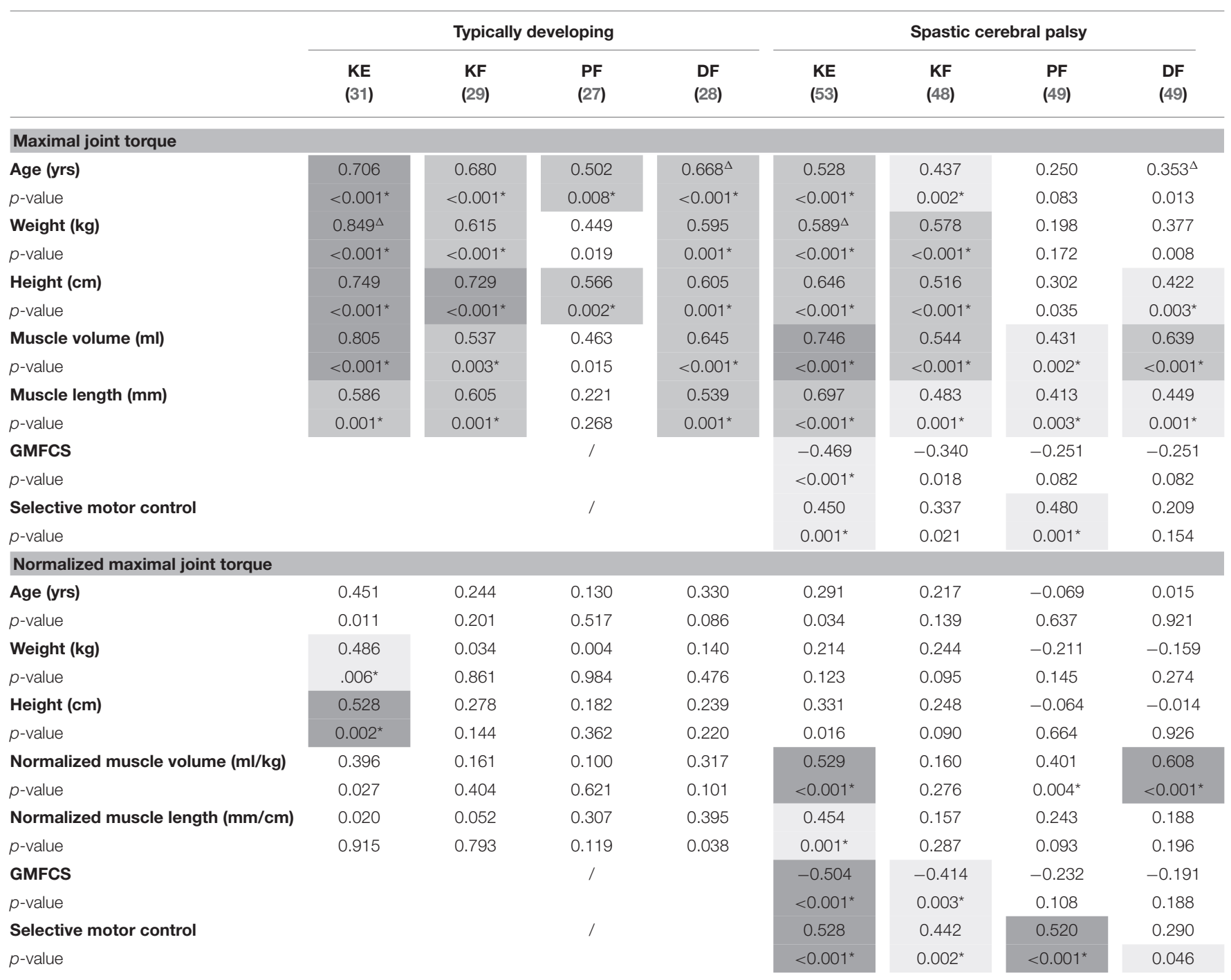

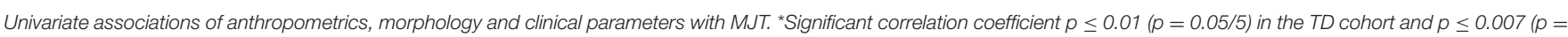
$0.05 / 7)$ in the SCP cohort. ${ }^{\Delta}$ Significant difference between the correlation coefficient in the two cohorts ( $\left.p \leq 0.05\right)$. The colors indicate the strength of the relationship: dark gray, high;

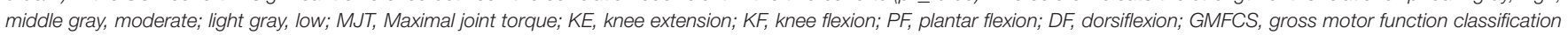
system.

most of the muscles in children with SCP are undersized, but even more underpowered, producing less force per unit muscle tissue.

\section{Relationships}

The second aim of the current study was to define the muscle size-strength relationship, as well as associations of anthropometric measures, GMFCS level, and SMC with muscle strength. The correlation coefficients of anthropometric parameters with MJT (Table 3) were similar between TD and SCP $(17,34,35,56)$. While the coefficients appeared a little lower in the SCP cohort, the only significantly different associations were for KE with weight $(Z=2.442)$ and for DF MJT with age $(Z=1.764)$. Similarly, the associations of muscle morphology parameters with MJT were comparable between the cohorts. This is in contrast with previous results by Reid et al. where correlation coefficients of MV with $\mathrm{KE}$ and $\mathrm{KF}$ isometric MJT were significantly lower in the SCP cohort. However, in the same investigation, they found no differences in the correlation coefficient of $\mathrm{MV}$ with the isokinetic joint torque or joint work (20).

Different conclusions could be drawn when MJT was normalized to body weight (Table 3 ). In the TD cohort, all correlation coefficients became non-significant, with an exception for KE nMJT with weight and height. These findings suggest that the variance in MJT is largely influenced by growthrelated parameters in this young, prepubertal TD cohort. In contrast to the TD group, the RF, MG, and TA nMV, as well as RF nML remained significantly associated with nMJT in the SCP cohort. These results confirmed that (muscular) growth is not the only crucial factor contributing to strength in children with SCP. This conclusion is further supported by the observed significant associations of SMC with MJT of KE and PF and nMJT of KE, 
TABLE 4 | Regressions best fit.

\begin{tabular}{|c|c|c|c|c|c|c|}
\hline Dependent variable & $\begin{array}{c}\text { Independent } \\
\text { variables }\end{array}$ & Adjusted $R^{2}$ & $p$-value & $\begin{array}{c}\text { Part } \\
\text { correlations }\end{array}$ & Tolerance & VIF \\
\hline \multicolumn{7}{|c|}{ A. Maximal joint torque in typically developing children } \\
\hline \multirow[t]{2}{*}{ Knee extension } & Height & 0.663 & $<0.001^{\star}$ & 0.232 & 0.248 & 4.038 \\
\hline & RF MV & & & 0.194 & 0.248 & 4.038 \\
\hline Knee flexion & Height & 0.514 & $<0.001^{\star}$ & 0.729 & - & - \\
\hline Plantar flexion & Height & 0.293 & $0.002^{\star}$ & 0.566 & - & - \\
\hline Dorsiflexion & TA MV & 0.393 & $<0.001^{*}$ & 0.645 & - & - \\
\hline \multicolumn{7}{|c|}{ B. Maximal joint torque in spastic cerebral palsy } \\
\hline Knee extension model 1 & RF MV & 0.548 & $<0.001^{\star}$ & 0.746 & - & - \\
\hline \multirow[t]{2}{*}{ Knee extension model 2} & RF MV & 0.601 & $<0.001^{\star}$ & 0.644 & 0.888 & 1.127 \\
\hline & SMC KE & & & 0.208 & 0.888 & 1.127 \\
\hline Knee flexion model 1 & ST MV & 0.281 & $<0.001^{\star}$ & 0.544 & - & - \\
\hline \multirow[t]{2}{*}{ Knee flexion model 2} & ST MV & 0.366 & $<0.001^{\star}$ & 0.529 & 0.988 & 1.012 \\
\hline & SMC KF & & & 0.276 & 0.988 & 1.012 \\
\hline Plantar flexion model 1 & MG MV & 0.168 & $0.002^{\star}$ & 0.431 & - & - \\
\hline \multirow[t]{2}{*}{ Plantar flexion model 2} & MG MV & 0.279 & $<0.001^{\star}$ & 0.280 & 0.882 & 1.113 \\
\hline & SMC PF & & & 0.355 & 0.882 & 1.113 \\
\hline Dorsiflexion model 1/2 & TA MV & 0.396 & $<0.001^{\star}$ & 0.639 & - & - \\
\hline \multicolumn{7}{|c|}{ C. Normalized maximal joint torque in spastic cerebral palsy } \\
\hline \multirow[t]{3}{*}{ Knee extension } & Height & 0.419 & $<0.001^{\star}$ & 0.206 & 0.947 & 1.056 \\
\hline & RF nMV & & & 0.312 & 0.788 & 1.269 \\
\hline & SMC KE & & & 0.326 & 0.825 & 1.213 \\
\hline \multirow[t]{2}{*}{ Knee flexion } & Height & 0.210 & $0.002^{\star}$ & 0.222 & 0.994 & 1.006 \\
\hline & SMC KF & & & 0.432 & 0.994 & 1.006 \\
\hline Plantar flexion & SMC PF & 0.254 & $<0.001^{\star}$ & 0.520 & - & - \\
\hline Dorsiflexion & TA nMV & 0.355 & $<0.001^{\star}$ & 0.607 & - & - \\
\hline
\end{tabular}

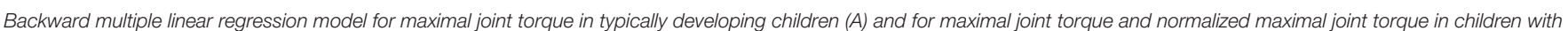

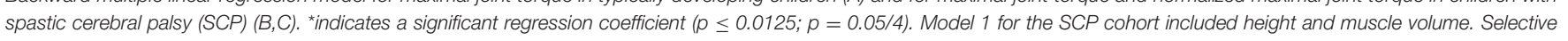

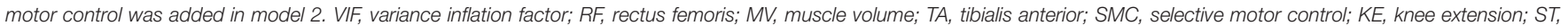
semitendinosus; KF, knee flexion; MG, medial gastrocnemius; PF, plantarflexion; $n M V$, normalized muscle volume.

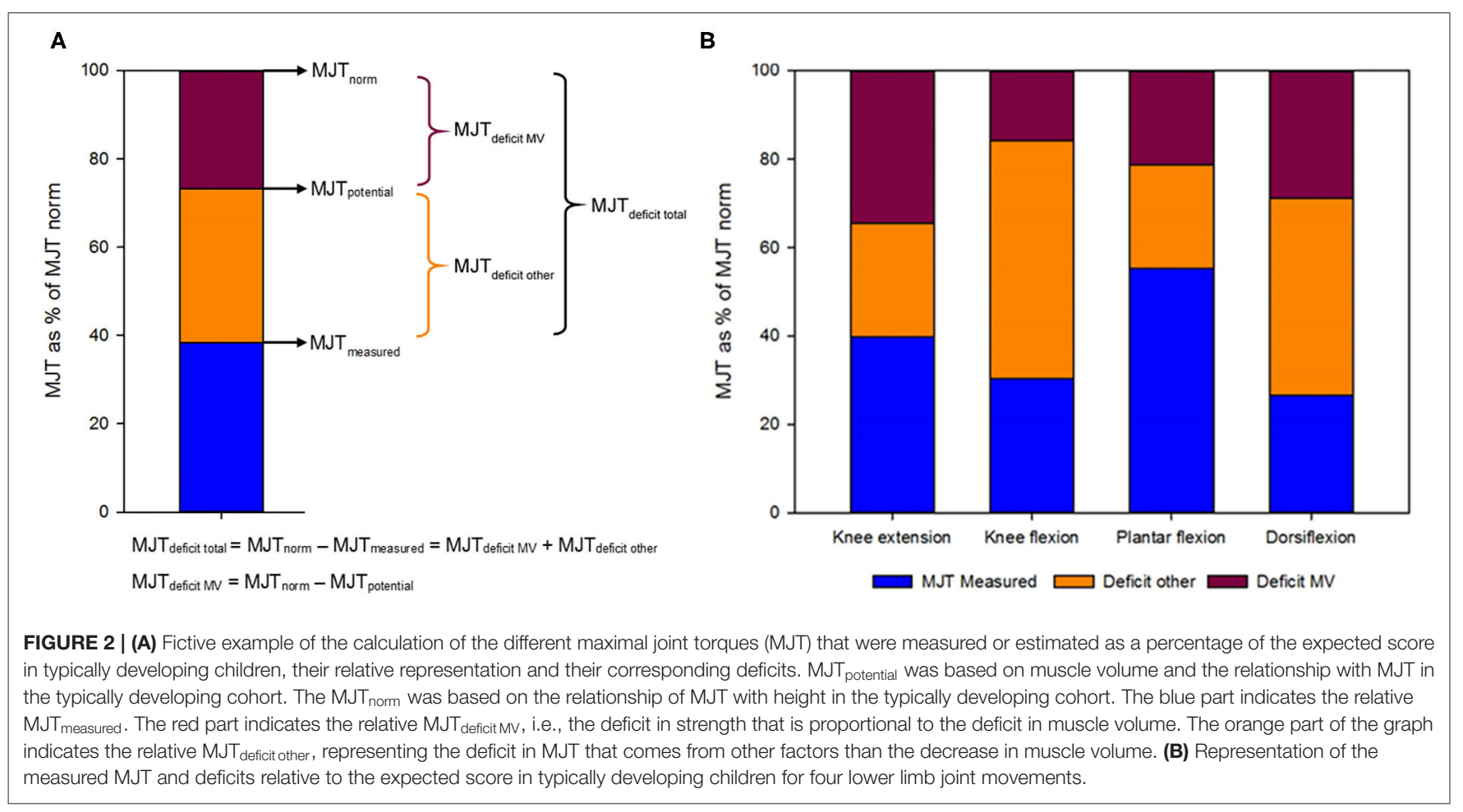


$\mathrm{KF}$, and PF, as well as GMFCS with MJT of KE and with nMJT of $\mathrm{KE}$ and KF. Earlier investigations found a similar influence of GMFCS level on both isometric and functional strength $(4,5$, $14,57)$. However, these results point toward the need for further research on the correct normalization parameters for muscle size and strength parameters in healthy and disabled pediatric populations. There was no significant negative association of nMJT with age in the CP cohort, indicating that the previously reported decrease in normalized muscle strength with age was not yet present in our group (16). This is presumably due to study differences in the age range of the cohort, which was 5-12 years in the current investigation in comparison to 8-19 years in the investigation by Davids et al.

In the multiple regression models for MJT in the TD group, height, MV, or both were included (Table 4A). Age, height, and weight as well as MV and ML showed high collinearity with each other (Supplementary Table 2), upon which only one parameter per category was chosen. The total explained variance of the TD models for MJT ranged from 29.3 to $66.3 \%$. This is lower than previous results for KE MJT from Moreau et al. (17), where muscle thickness of the vastus lateralis and age resulted in an explained variance of $91 \%$. Yet, the latter study was performed in a small study sample $(N=12)$ with a much wider age range (7-20 years) than the current study sample. The first multiple regression model of MJT in the SCP cohort included MV for all four joint movements, resulting in explained variances ranging from 16.8 to $54.8 \%$ (Table $4 \mathrm{~B}$ ). Adding SMC to the model increased the explained variance for $\mathrm{KE}, \mathrm{KF}$, and $\mathrm{PF}$ by $5.3-$ $11.1 \%$, resulting in a range of $27.9-60.1 \%$ explained variance. Likewise, SMC was included in the models for nMJT for KE, $\mathrm{KF}$, and PF (Table 4C). SMC has, to our knowledge, not yet been related to isometric strength. However, SMC as assessed in this investigation has been found to be significantly related to gait parameters around the ankle (24). Moreover, both the Gross Motor Function Measure and overall gait deviation have been found significantly associated with another selectivity score, i.e., the Selective Control Assessment of the Lower Extremity (SCALE) $(12,13)$.

\section{Contributions to Muscle Weakness}

The final aim of this investigation was to define the contribution of decreased muscle size to the deficits in muscle strength. The group results for the four joint movements indicated that the patterns in the proportion of muscle weakness explained by decreased MV and by other factors are muscle specific (Figure 2 and Supplementary Table 3). The part explained by $\mathrm{MV}$, i.e., the difference between the $\mathrm{MJT}_{\text {norm }}$ based on growth and the $\mathrm{MJT}_{\text {potential }}$ based on MV, ranged between $22.6 \%$ for $\mathrm{KF}$ and $57.3 \%$ for KE. The part explained by other factors, i.e., the difference between the measured MJT and $\mathrm{MJT}_{\text {potential, }}$, ranged from $42.7 \%$ for $\mathrm{KE}$ to $77.4 \%$ for KF. The weakness in $\mathrm{KE}$ and PF presented with approximately equal distributions of $\mathrm{MV}$ deficits and remaining factors, whereas $\mathrm{KF}$ and $\mathrm{DF}$ muscle weakness seemed predominantly caused by other factors. This variability between joint movements can potentially be partially explained by the differences in architectural types of the investigated muscles (like the fascicle arrangement), as well as by the differences in neural control and common treatment at the muscular level. Additionally, muscle weakness, as well as the contributing factors to muscle weakness, largely varied between participants (Supplementary Figure 1). This may be caused by participant-specific characteristics, like cognitive ability and motivation, as well as by the treatment history with, among others, the focus of regular physiotherapy, the use of orthoses, and the number of botulinum neurotoxin-A injections $(44,58$, 59).

There may be several additional underlying mechanisms, the "other factors," causing the disproportional decrease in muscle strength. A simple, clinical measure of SMC was used to represent the neural component in this investigation and found to have moderately significant associations with MJT of KE, KF, and PF (Table 3). The lack of studies evaluating the associations of SMC with isometric muscle strength in previous investigations made it difficult to compare results. However, various underlying neurological factors influencing muscle strength have been identified like reduced central drive, impaired reciprocal inhibition, and disorganized motor unit recruitment $(6,7)$. Moreover, it should be noted that there is also individual variation in neural control of muscle activation in the healthy population (60), which was confirmed by the wide spread of the TD data around the regression line in Figure 1. Furthermore, the assessment of strength can also be influenced by cognition, attention, or motivation (17). So far, previous studies on the role of muscle selectivity, using the SCALE outcome (61), only reported the total limb score without description of the individual scores. Moreover, the SCALE evaluates both directions of movement around one joint in one score (e.g., KF and KE), instead of separately per motion direction, as applied in the current study.

Next to the alterations in neural control, additional muscular changes may also be considered as underlying mechanism of the decreased muscle strength. Muscle architecture parameters, such as the pennation angle and fascicle length, are related to the pCSA and, therefore, to maximal strength. Yet, previous investigations found inconsistent outcomes for fascicle length in participants with SCP $(23,29)$. Additionally, the ML-force relationship, also known as the torque-angle relationship, is considered to be altered in children with SCP, resulting in measurements being performed at different points of the lengthforce curve in the TD cohort vs. the SCP cohort $(62,63)$. Earlier research also indicated alterations in the proportion of contractile tissue relative to non-contractile (fibrotic and fatty) tissue in children with SCP. It is likely that this reduced proportion of contractile tissue in the already reduced $\mathrm{MV}$ in children with SCP, compared to their TD peers, also contributes to the observed muscle weakness $(21,22,64)$. Yet, individual variation in muscle tissue composition has been determined in the healthy population (65). Future investigations are needed to further delineate and understand the contributions of these "other factors" to muscle weakness in SCP, with a systematic focus on the neuromuscular control, the muscle architecture, the length-force relationship, and the intrinsic muscle composition. Since muscle strength is an important parameter for gross motor function and maintaining ambulation, further research into the 
underlying components of muscle weakness is encouraged (15, $16,36)$.

\section{Clinical Implications}

The muscle strength profile resulting from the combined assessment of muscle strength and muscle morphology gives an indication of the contribution of muscle size deficits to muscle weakness. This could be used to optimize training prescriptions either aiming at enhancing neural drive or inducing muscle hypertrophy. However, for both underlying mechanisms of muscle weakness, the influence of training is not always consistent $(44,66,67)$. The muscle strength profile could potentially provide a predictive value on the outcomes of certain types of strength or active movement training in children with SCP. Knowing the contribution of muscle size to muscle weakness could also be used to define if other, potentially atrophy-inducing, treatments like botulinum neurotoxin-A or lower leg casting (68-71) are appropriate for a patient or a specific muscle group.

Impaired muscle growth and muscle size deficits are also underlying causes for muscle contractures in children with SCP $(72,73)$. Muscle contractures can be defined as unique muscular adaptations that increase the passive stiffness of the muscle, resulting in limited mobility of the joints without active force production of the muscle (74). Reduced muscle growth, as already observed from the age of 15 months (34), may result in reduced $\mathrm{ML}$ and muscle-tendon unit length relative to bone length. Although shortened fascicle lengths, related to the number of sarcomeres in series, can be a possible reason for decreased ML (75), this has not been confirmed in every investigation (76). However, in pennate muscles, both the length and the diameter of the fascicle contribute to ML (77). Consequently, reduced MV resulting from reduced muscle fiber diameter, and related to the number of sarcomeres in parallel, can influence longitudinal muscle growth $(77,78)$. The contribution of reduced muscle diameter to reduced ML depends on the morphology of the muscle and fascicle arrangement. Future studies should define the impact of muscle size deficits on muscle contractures and define the influence of common interventions like stretching and casting.

\section{Limitations and Future Perspectives}

There were some limitations to this investigation that should be considered when interpreting the results. First, there was an unequal distribution of GMFCS levels, with a multitude of children classified as GMFCS level I. This was influenced by the selected inclusion criteria related to previous treatment history and the ability to cognitively understand the test procedures, since orthopedic surgeries and cognitive problems are more common in children with higher GMFCS levels (79-82). This investigation had a cross-sectional design limited to prepubertal children. Future investigations should consider longitudinal follow-up to define the alterations of muscle size, strength, and their ratios during growth and aging, as well as the effect of interventions to prevent or improve muscle weakness. This might also uncover the timing of neural and musculoskeletal onset as causes of muscle weakness. Finally, this investigation applied some simplifications. The morphology was only assessed for one muscle per joint movement. Previous investigations showed that all muscles of the lower limb in children with SCP are affected. However, there is heterogeneity, and not all muscles are affected to the same extent $(25,28,83)$. Moreover, a subjective clinical classification of SMC was included. Further research into the underlying neural components of muscle weakness is encouraged.

\section{CONCLUSION}

This investigation confirmed the disproportional decreases in muscle size and muscle strength around the knee and ankle joint of children with SCP in comparison to TD children. Furthermore, associations of strength with growth-related parameters like age, weight, and height were strongest in the TD cohort, whereas these were also present but accompanied by associations with SMC and GMFCS in the SCP cohort. The correlation coefficients of the muscle size-strength relationship were similar, whereas the regression coefficient was decreased in the SCP cohort, indicating that only part of the muscle weakness can be attributed to smaller MVs. However, there was a lot of heterogeneity between the proportion of muscle weakness that was attributed to deficits in muscle size both between joint movements and between subjects. Future studies should investigate what other mechanisms underlie muscle weakness, as well as how muscle weakness and its components are influenced by treatment, growth, and aging.

\section{DATA AVAILABILITY STATEMENT}

The raw data supporting the conclusions of this article will be made available by the authors, without undue reservation.

\section{ETHICS STATEMENT}

The studies involving human participants were reviewed and approved by Ethical Committee of the University Hospitals Leuven and Ghent. Written informed consent to participate in this study was provided by the participants' legal guardian/next of kin.

\section{AUTHOR CONTRIBUTIONS}

This study was designed by $\mathrm{BH}, \mathrm{KD}, \mathrm{CV}, \mathrm{PC}, \mathrm{MD}$, and LB-O. $\mathrm{BH}, \mathrm{NP}, \mathrm{IV}$, and NDB contributed to the data collection. AV and $\mathrm{GM}$ were involved in patient recruitment. $\mathrm{BH}$ was responsible for the data processing, with help from IV in the MATLAB coding and conducted all presented analyses. $\mathrm{BH}$ and $\mathrm{KD}$ contributed to the interpretation of the results and were involved in the critical revision and editing of the manuscript that was written by $\mathrm{BH}$. All authors approved the final version of the manuscript and agreed to be accountable for the content of the work. 


\section{FUNDING}

BH and NP were funded by a TBM grant (TAMTA-T005416N). This work was further supported by Internal Funds of KU Leuven (C24/18/103), the Flemish Organisation for Scientific Research (3M180752), and the Duchenne Parent Project NL (17.011). IV was funded by a doctoral grant from the Flemish Organisation for Scientific Research (FWO-1188921N) and LB-O from the Dutch Organisation for Scientific Research (NWO-016.186.144).

\section{ACKNOWLEDGMENTS}

The authors would like to thank Francesco Cenni and SimonHenri Schless for the development of the 3DfUS technique, as well as Marije Goudriaan for the development of the customdesigned chair in which isometric strength was assessed. The

\section{REFERENCES}

1. Rosenbaum P, Paneth N, Leviton A, Goldstein M, Bax M, Damiano D, et al. A report: the definition and classification of cerebral palsy April 2006. Dev Med Child Neurol. (2007) 49:8-14. doi: 10.1111/j.1469-8749.2007.tb12610.x

2. Surveillance of Cerebral Palsy in Europe. Prevalence and characteristics of children with cerebral palsy in Europe. Dev Med Child Neurol. (2002) 44:63340. doi: 10.1111/j.1469-8749.2002.tb00848.x

3. Graham HK, Rosenbaum P, Paneth N, Dan B, Lin J-P, Damiano DL, et al. Cerebral palsy. Nat Rev Dis Prim. (2016) 7:15082. doi: 10.1038/nrdp.2016.5

4. Wiley ME, Damiano DL. Lower-extremity strength profiles in spastic cerebral palsy. Dev Med Child Neurol. (1998) 40:1007. doi: 10.1111/j.1469-8749.1998.tb15369.x

5. Goudriaan M, Nieuwenhuys A, Schless SH, Goemans N, Molenaers G, Desloovere K. A new strength assessment to evaluate the association between muscle weakness and gait pathology in children with cerebral palsy. PLoS ONE. (2018) 13:e0191097. doi: 10.1371/journal.pone.0191097

6. Elder GCB, Kirk J, Stewart G, Cook K, Weir D, Marshall A, et al. Contributing factors to muscle weakness in children with cerebral palsy. Dev Med Child Neurol. (2003) 45:542-50. doi: 10.1017/S00121622030 00999

7. Mockford M, Caulton JM. The pathophysiological basis of weakness in children with cerebral palsy. Pediatr Phys Ther. (2010) 22:22233. doi: 10.1097/PEP.0b013e3181dbaf96

8. Edwards RHT. Physiological analysis of skeletal muscle weakness and fatigue. Clin Sci Mol Med. (1978) 54:463-70. doi: 10.1042/cs0540463

9. Desloovere K, Molenaers G, Feys H, Huenaerts C, Callewaert B, Walle P Van de. Do dynamic and static clinical measurements correlate with gait analysis parameters in children with cerebral palsy? Gait Posture. (2006) 24:302-13. doi: 10.1016/j.gaitpost.2005.10.008

10. Ross SA, Engsberg JR. Relationships between spasticity, strength, gait, and the GMFM-66 in persons with spastic diplegia cerebral palsy. Arch Phys Med Rehabil. (2007) 88:1114-20. doi: 10.1016/j.apmr.2007.06.011

11. Kim WH, Park EY. Causal relation between spasticity, strength, gross motor function, and functional outcome in children with cerebral palsy: a path analysis. Dev Med Child Neurol. (2011) 53:68-73. doi: 10.1111/j.1469-8749.2010.03777.x

12. Noble JJ, Gough M, Shortland AP. Selective motor control and gross motor function in bilateral spastic cerebral palsy. Dev Med Child Neurol. (2019) 61:57-61. doi: 10.1111/dmcn.14024

13. Chruscikowski E, Fry NRD, Noble JJ, Gough M, Shortland AP. Selective motor control correlates with gait abnormality in children with cerebral palsy. Gait Posture. (2017) 52:107-9. doi: 10.1016/j.gaitpost.2016.11.031

14. Eek MN, Beckung E. Walking ability is related to muscle strength in children with cerebral palsy. Gait Posture. (2008) 28:366-71. doi: 10.1016/j.gaitpost.2008.05.004 authors are also very grateful to all the participants who participated in this study and their parents.

\section{SUPPLEMENTARY MATERIAL}

The Supplementary Material for this article can be found online at: https://www.frontiersin.org/articles/10.3389/fneur. 2021.692582/full\#supplementary-material

Supplementary Figure 1 | Display of the measured and estimated maximal joint torques (MJT)s per participant. The blue bar indicates the MJT measured, the red part is the deficit to $\mathrm{MJT}_{\text {norm }}$ due to decreased muscle volume (MV,) and the orange part of the graph indicates the deficit in MJT that comes from other factors than the decrease in MV. A negative red bar indicates an MJTpotential based on MV that is larger than expected from growth. A negative orange bar indicates an MJT $_{\text {measured }}$ that is larger than expected based on MV. For all four joint movements, the bars on the $\mathrm{x}$-axis are ranked from smallest to tallest child (in height), resulting in the same order of children for all four graphs.
15. Moreau NG. Muscle performance in children and youth with cerebral palsy: implications for resistance training. Cereb Palsy. (2020) 2629-40. doi: 10.1007/978-3-319-74558-9_164

16. Davids JR, Oeffinger DJ, Bagley AM, Sison-Williamson M, Gorton G. Relationship of strength, weight, age, and function in ambulatory children with cerebral palsy. J Pediatr Orthop. (2014) 35:523-9. doi: 10.1097/BPO.0000000000000320

17. Moreau NG, Simpson KN, Teefey SA, Damiano DL, Moreau NG. Muscle architecture predicts maximum strength and is related to activity levels in cerebral palsy. Phys Ther. (2010) 90:1619-30. doi: 10.2522/ptj.20090377

18. Hussain AW, Onambele GL, Williams AG, Morse CI. Muscle size, activation, and coactivation in adults with cerebral palsy. Muscle Nerve. (2014) 49:7683. doi: $10.1002 /$ mus. 23866

19. Stackhouse SK, Binder-Macleod SA, Lee SCK. Voluntary muscle activation, contractile properties, and fatigability in children with and without cerebral palsy. Muscle Nerve. (2005) 31:594-601. doi: 10.1002/mus.20302

20. Reid SL, Pitcher CA, Williams SA, Licari MK, Valentine JP, Shipman PJ, et al. Does muscle size matter? The relationship between muscle size and strength in children with cerebral palsy. Disabil Rehabil. (2015) 37:57984. doi: 10.3109/09638288.2014.935492

21. Noble JJ, Charles-Edwards GD, Keevil SF, Lewis AP, Gough M, Shortland AP. Intramuscular fat in ambulant young adults with bilateral spastic cerebral palsy. BMC Musculoskelet Disord. (2014) 15:236. doi: 10.1186/1471-2474-15-236

22. Young HJ, Jenkins NT, Zhao Q, Mccully KK. Measurement of intramuscular fat by muscle echo intensity. Muscle and Nerve. (2015) 52:963-71. doi: 10.1002/mus.24656

23. Williams SA, Stott NS, Valentine J, Elliott C, Reid SL. Measuring skeletal muscle morphology and architecture with imaging modalities in children with cerebral palsy: a scoping review. Dev Med Child Neurol. (2020) 63:111. doi: $10.1111 / \mathrm{dmcn} .14714$

24. Schless SH, Cenni F, Bar-On L, Hanssen B, Goudriaan M, Papageorgiou E, et al. Combining muscle morphology and neuromotor symptoms to explain abnormal gait at the ankle joint level in cerebral palsy. Gait Posture. (2019) 68:531-7. doi: 10.1016/j.gaitpost.2018.12.002

25. Noble JJ, Fry NR, Lewis AP, Keevil SF, Gough M, Shortland AP. Lower limb muscle volumes in bilateral spastic cerebral palsy. Brain Dev. (2014) 36:294-300. doi: 10.1016/j.braindev.2013.05.008

26. Massaad A, Assi A, Bakouny Z, Bizdikian AJ, Skalli W, Ghanem I. Alterations of treatment-naïve pelvis and thigh muscle morphology in children with cerebral palsy. J Biomech. (2019) 82:178-85. doi: 10.1016/j.jbiomech.2018.10.022

27. Barber L, Hastings-Ison T, Baker R, Barrett R, Lichtwark G. Medial gastrocnemius muscle volume and fascicle length in children aged 2 to 5years with cerebral palsy. Dev Med Child Neurol. (2011) 53:5438. doi: 10.1111/j.1469-8749.2011.03913.x 
28. Handsfield GG, Meyer CH, Abel MF, Blemker SS. Heterogeneity of muscle sizes in the lower limbs of children with cerebral palsy. Muscle and Nerve. (2016) 53:933-45. doi: 10.1002/mus.24972

29. Barrett RS, Lichtwark GA. Gross muscle morphology and structure in spastic cerebral palsy: a systematic review. Dev Med Child Neurol. (2010) 52:794804. doi: $10.1111 /$ j.1469-8749.2010.03686.x

30. Moreau NG, Teefey SA, Damiano DL. In vivo muscle architecture and size of the rectus femoris and vastus lateralis in children and adolescents with cerebral palsy. Dev Med Child Neurol. (2010) 51:8006. doi: 10.1111/j.1469-8749.2009.03307.x

31. Haberfehlner H, Jaspers RT, Rutz E, Becher JG, Harlaar J, van der Sluijs JA, et al. Knee moment-angle characteristics and semitendinosus muscle morphology in children with spastic paresis selected for medial hamstring lengthening. PLoS ONE. (2016) 11:e0166401. doi: 10.1371/journal.pone.0166401

32. Schless S-H, Hanssen B, Cenni F, Bar-On L, Aertbelien E, Molenaers G, et al. Estimating medial gastrocnemius muscle volume in children with spastic cerebral palsy: a cross-sectional investigation. Dmcn. (2017) 60:17. doi: $10.1111 / \mathrm{dmcn} .13597$

33. Mohagheghi AA, Khan T, Meadows TH, Giannikas K, Baltzopoulos V, Maganaris CN. Differences in gastrocnemius muscle architecture between the paretic and non-paretic legs in children with hemiplegic cerebral palsy. Clin Biomech. (2007) 22:718-24. doi: 10.1016/j.clinbiomech.2007.03.004

34. Herskind A, Ritterband-Rosenbaum A, Willerslev-Olsen M, Lorentzen J, Hanson L, Lichtwark G, et al. Muscle growth is reduced in 15-monthold children with cerebral palsy. Dev Med Child Neurol. (2016) 58:48591. doi: $10.1111 / \mathrm{dmcn} .12950$

35. Willerslev-Olsen M, Lund MC, Lorentzen J, Barber L, Kofoed-Hansen M, Nielsen JB. Impaired muscle growth precedes development of increased stiffness of the triceps surae musculotendinous unit in children with cerebral palsy. Dev Med Child Neurol. (2018) 60:672-9. doi: 10.1111/dmcn.13729

36. Shortland A. Muscle deficits in cerebral palsy and early loss of mobility: can we learn something from our elders? Dev Med Child Neurol. (2009) 51:59-63. doi: 10.1111/j.1469-8749.2009.03434.x

37. Ohata K, Tsuboyama T, Haruta T, Ichihashi N, Kato T, Nakamura T. Relation between muscle thickness, spasticity, and activity limitations in children and adolescents with cerebral palsy. Dev Med Child Neurol. (2008) 50:1526. doi: 10.1111/j.1469-8749.2007.02018.x

38. Ohata K, Tsuboyama $\mathrm{T}$, Haruta $\mathrm{T}$, Ichihashi $\mathrm{N}$, Nakamura $\mathrm{T}$. Longitudinal change in muscle and fat thickness in children and adolescents with cerebral palsy. Dev Med Child Neurol. (2009) 51:943-8. doi: 10.1111/j.1469-8749.2009.03342.x

39. Choe YR, Kim JS, Kim KH, Yi TI. Relationship between functional level and muscle thickness in young children with cerebral palsy. Ann Rehabil Med. (2018) 42:286. doi: 10.5535/arm.2018.42.2.286

40. Akagi R, Takai Y, Ohta M, Kanehisa H, Kawakami Y, Fukunaga T. Muscle volume compared to cross-sectional area is more appropriate for evaluating muscle strength in young and elderly individuals. Age Ageing. (2009) 38:5649. doi: 10.1093/ageing/afp122

41. Pitcher CA, Elliott CM, Williams SA, Licari MK, Kuenzel A, Shipman PJ, et al. Childhood muscle morphology and strength: alterations over six months of growth. Muscle Nerve. (2012) 46:360-6. doi: 10.1002/mus.23326

42. Fukunaga T, Miyatani M, Tachi M, Kouzaki M, Kawakami Y. Muscle volume is a major determinant of joint torque in humans. Acta Physiol Scand. (2001) 172:249-55. doi: 10.1046/j.1365-201x.2001.00867.x

43. Franki I, Molenaers LBG, Campenhout A Van, Craenen K, Desloovere K, Feys $\mathrm{H}$, et al. Tone reduction and physical therapy: strengthening partners in treatment of children with spastic cerebral palsy. Neuropediatrics. (2020) 51:89-104. doi: 10.1055/s-0039-3400987

44. Walhain F, Desloovere K, Declerck M, Van Campenhout A, Bar-On L. Interventions and lower-limb macroscopic muscle morphology in children with spastic cerebral palsy: a scoping review. Dev Med Child Neurol. (2020) 63:1-13. doi: $10.1111 /$ dmcn. 14652

45. Palisano RJ, Rosenbaum P, Bartlett D, Livingston MH. Content validity of the expanded and revised Gross Motor Function Classification System. Dev Med Child Neurol. (2008) 50:744-50. doi: 10.1111/j.1469-8749.2008.03089.x

46. Bohannon RW, Smith MB. Inter rater reliability of a modified Ashworth Scale of muscle spasticity. Phys Ther. (1987) 67:206-7. doi: 10.1093/ptj/67.2.206
47. Boyd R. Objective measurement of clinical findings in the use of botulinum toxin type A for the management of children with cerebral palsy. Eur J Neurol. (1999) 6:1-13. doi: 10.1111/j.1468-1331.1999.tb00031.x

48. Matthews WB. Aids to the examination of the peripheral nervous system. $J$ Neurol Sci. (1977) 33:299. doi: 10.1016/0022-510X(77)90205-2

49. Gage JR, Schwartz M, Koop S, Novacheck T. The Identification and Treatment of Gait Problems in Cerebral Palsy. London: Mac Keith Press (2009). 187 p.

50. Cenni F, Monari D, Desloovere K, Erwin A, Schless S, Bruyninckx $\mathrm{H}$. The reliability and validity of a clinical 3D freehand ultrasound system. Comput Methods Programs Biomed. (2016) 136:179-87. doi: 10.1016/j.cmpb.2016.09.001

51. Cenni F, Schless S-H, Bar-On L, Molenaers G, Van Campenhout A, Aertbeliën E, et al. Can in vivo medial gastrocnemius muscle-tendon unit lengths be reliably estimated by two ultrasonography methods? A within-session analysis. Ultrasound Med Biol. (2018) 44:110-8. doi: 10.1016/j.ultrasmedbio.2017.09.018

52. Barber L, Alexander C, Shipman P, Boyd R, Reid S, Elliott C. Validity and reliability of a freehand 3D ultrasound system for the determination of triceps surae muscle volume in children with cerebral palsy. J Anat. (2018) 234:384-91. doi: 10.1111/joa.12927

53. Hanssen B, De Beukelaer N, Schless S-H, Cenni F, Bar-On L, Peeters $\mathrm{N}$, et al. Reliability of processing 3-D freehand ultrasound data to define muscle volume and echo-intensity in pediatric lower limb muscles with typical development or with spasticity. Ultrasound Med Biol. (2021). doi: 10.1016/j.ultrasmedbio.2021.04.028. [Epub ahead of print].

54. Hinkle D, Wiersma W, Jurs S. Applied Statistics for the Behavioural Sciences. Boston, MA: Houghton Mifflin (2003).

55. O'Brien SM, Carroll TJ, Barber LA, Lichtwark GA. Plantar flexor voluntary activation capacity, strength and function in cerebral palsy. Eur J Appl Physiol. (2021). doi: 10.1007/s00421-021-04638-z

56. Obst SJ, Bickell R, Florance K, Boyd RN, Read F, Barber L. The size and echogenicity of the tibialis anterior muscle is preserved in both limbs in young children with unilateral spastic cerebral palsy. Disabil Rehabil. (2020) 43:1-10. doi: 10.1080/09638288.2020.1863482

57. Gillett JG, Lichtwark GA, Boyd RN, Barber LA. Functional capacity in adults with cerebral palsy: lower limb muscle strength matters. Arch Phys Med Rehabil. (2018) 99:900-6. doi: 10.1016/j.apmr.2018.01.020

58. Hastings-Ison T, Graham HK. Atrophy and hypertrophy following injections of botulinum toxin in children with cerebral palsy. Dev Med Child Neurol. (2013) 55:778-9. doi: 10.1111/dmcn.12231

59. Schless SH, Cenni F, Bar-On L, Hanssen B, Kalkman B, O’brien T, et al. Medial gastrocnemius volume and echo-intensity after botulinum neurotoxin A interventions in children with spastic cerebral palsy. Dev Med Child Neurol. (2019) 61:783-90. doi: 10.1111/dmcn.14056

60. Belanger AY, Mccomas AJ. Extent of motor unit activiation during effort. $J$ Appl. (1981) 51:1131-5. doi: 10.1152/jappl.1981.51.5.1131

61. Fowler EG, Staudt LA, Greenberg MB, Oppenheim WL. Selective Control Assessment of the Lower Extremity (SCALE): development, validation, and interrater reliability of a clinical tool for patients with cerebral palsy. Dev Med Child Neurol. (2009) 51:607-14. doi: 10.1111/j.1469-8749.2008.03186.x

62. Lieber RL, Friden J. Functional and clinical significance of skeletal muscle architecture. Muscle Nerve. (2000) 23:1647-66. doi: 10.1002/10974598(200011)23:11<1647::AID-MUS1>3.0.CO;2-M

63. Frisk RF, Lorentzen J, Barber L, Nielsen JB. Characterization of torque generating properties of ankle plantar flexor muscles in ambulant adults with cerebral palsy. Eur J Appl Physiol. (2019) 119:1127-36. doi: 10.1007/s00421-019-04102-z

64. Obst SI, Boyd R, Read F, Barber L. Quantitative 3-D ultrasound of the medial gastrocnemius muscle in children with unilateral spastic cerebral palsy. Ultrasound Med Biol. (2017) 43:2814-23. doi: 10.1016/j.ultrasmedbio.2017.08.929

65. Jones EJ, Bishop PA, Woods AK, Green JM. Cross-sectional area and muscular strength: a brief review. Sport Med. (2008) 38:98794. doi: 10.2165/00007256-200838120-00003

66. Gillett JG, Boyd RN, Carty CP, Barber LA. The impact of strength training on skeletal muscle morphology and architecture in children and adolescents with spastic cerebral palsy: a systematic review. Res Dev Disabil. (2016) 56:183-96. doi: 10.1016/j.ridd.2016.06.003 
67. O'Brien SM, Lichtwark GA, Carroll TJ, Barber LA. Impact of lower limb active movement training in individuals with spastic type cerebral palsy on neuromuscular control outcomes: a systematic review. Front Neurol. (2020) 11:581892. doi: 10.3389/fneur.2020.581892

68. Clark BC. In vivo alterations in skeletal muscle form and function after disuse atrophy. Med Sci Sports Exerc. (2009) 41:1869-75. doi: 10.1249/MSS.0b013e3181a645a6

69. Rooks DS, Laurent D, Praestgaard J, Rasmussen S, Bartlett M, Tankó LB. Effect of bimagrumab on thigh muscle volume and composition in men with casting-induced atrophy. J Cachexia Sarcopenia Muscle. (2017) 8:72734. doi: 10.1002/jcsm.12205

70. Gough M, Fairhurst C, Shortland a P. Botulinum toxin and cerebral palsy: time for reflection? Dev Med Child Neurol. (2005) 47:70912. doi: $10.1017 /$ S0012162205001453

71. Schroeder AS, Ertl-Wagner B, Britsch S, Schröder JM, Nikolin S, Weis J, et al. Muscle biopsy substantiates long-term MRI alterations one year after a single dose of botulinum toxin injected into the lateral gastrocnemius muscle of healthy volunteers. Mov Disord. (2009) 24:1494-503. doi: 10.1002/mds.22661

72. Pingel J, Bartels EM, Nielsen JB. New perspectives on the development of muscle contractures following central motor lesions. J Physiol. (2017) 595:1027-38. doi: 10.1113/JP272767

73. Gough M, Shortland AP. Could muscle deformity in children with spastic cerebral palsy be related to an impairment of muscle growth and altered adaptation? Dev Med Child Neurol. (2012) 54:495-9. doi: 10.1111/j.1469-8749.2012.04229.x

74. Smith LR, Lee KS, Ward SR, Chambers HG, Lieber RL. Hamstring contractures in children with spastic cerebral palsy result from a stiffer extracellular matrix and increased in vivo sarcomere length. J Physiol. (2011) 589:2625-39. doi: 10.1113/jphysiol.2010.203364

75. Matthiasdottir S, Hahn M, Yaraskavitch M, Herzog W. Muscle and fascicle excursion in children with cerebral palsy. Clin Biomech. (2014) 29:458-62. doi: 10.1016/j.clinbiomech.2014.01.002

76. Mathewson MA, Ward SR, Chambers HG, Lieber RL. High resolution muscle measurements provide insights into equinus contractures in patients with cerebral palsy. J Orthop Res. (2015) 33:33-9. doi: 10.1002/jor. 22728

77. Weide G, Huijing PA, Maas JC, Becher JG, Harlaar J, Jaspers RT. Medial gastrocnemius muscle growth during adolescence is mediated by increased fascicle diameter rather than by longitudinal fascicle growth. J Anat. (2015) 226:530-41. doi: 10.1111/joa.12306
78. Bénard MR, Harlaar J, Becher JG, Huijing PA, Jaspers RT. Effects of growth on geometry of gastrocnemius muscle in children: a three-dimensional ultrasound analysis. J Anat. (2011) 219:388-402. doi: 10.1111/j.1469-7580.2011.01402.x

79. Soo B, Howard JJ, Boyd RN, Reid SM, Lanigan A, Wolfe R, et al. Hip displacement in cerebral palsy. J Bone Jt Surg Ser A. (2006) 88:1219. doi: 10.2106/00004623-200601000-00015

80. Rehbein I, Teske V, Pagano I, Cúneo A, Pérez ME, von Heideken J. Analysis of orthopedic surgical procedures in children with cerebral palsy. World $J$ Orthop. (2020) 11:222-31. doi: 10.5312/wjo.v11.i4.222

81. Stadskleiv K, Jahnsen R, Andersen GL, von Tetzchner S. Neuropsychological profiles of children with cerebral palsy. Dev Neurorehabil. (2018) 21:108-20. doi: 10.1080/17518423.2017.12 82054

82. Stadskleiv K. Cognitive functioning in children with cerebral palsy. Dev Med Child Neurol. (2020) 62:283-9. doi: 10.1111/dmcn.14463

83. Handsfield GG, Meyer CH, Hart JM, Abel MF, Blemker SS. Relationships of 35 lower limb muscles to height and body mass quantified using MRI. J Biomech. (2014) 47:631-8. doi: 10.1016/j.jbiomech.2013. 12.002

Conflict of Interest: The authors declare that the research was conducted in the absence of any commercial or financial relationships that could be construed as a potential conflict of interest.

Publisher's Note: All claims expressed in this article are solely those of the authors and do not necessarily represent those of their affiliated organizations, or those of the publisher, the editors and the reviewers. Any product that may be evaluated in this article, or claim that may be made by its manufacturer, is not guaranteed or endorsed by the publisher.

Copyright (C) 2021 Hanssen, Peeters, Vandekerckhove, De Beukelaer, Bar-On, Molenaers, Van Campenhout, Degelaen, Van den Broeck, Calders and Desloovere. This is an open-access article distributed under the terms of the Creative Commons Attribution License (CC BY). The use, distribution or reproduction in other forums is permitted, provided the original author(s) and the copyright owner(s) are credited and that the original publication in this journal is cited, in accordance with accepted academic practice. No use, distribution or reproduction is permitted which does not comply with these terms. 